\title{
CARACTERIZAÇÃO SENSORIAL DO ODOR DA Aphanothece microscopic Nägeli DESIDRATADA
}

\author{
MARIA ISABEL QUEIROZ * \\ ROSA DE OLIVEIRA TREPTOW ** \\ PAULO ROBERTO KOETZ *
}

\begin{abstract}
Caracterizou-se o atributo odor da Aphanothece microscopic Nägeli visando sua utilização na alimentação humana. Levantou-se a terminologia para descrição do seu odor, assim como da Spirulina $s p$ e de uma alga comercial, a partir de consumidores. Selecionou-se e treinou-se equipe para expressar a intensidade do odor, de acordo com padrões (penetrante, ervas, ração e produtos do mar) obtidos a partir da terminologia descrita pelos consumidores. Os resultados obtidos indicam que tanto a Aphanothece microscopic Nägeli, como a Spirulina $s p$ e a alga comercial japonesa apresentaram odor penetrante, associado em primeiro lugar com ervas, em segundo com odor de ração e finalmente com odor de produtos do mar. A Aphanothece microscopic Nägeli apresenta odor característico, moderadamente forte a ervas e ligeiramente penetrante.
\end{abstract}

\section{INTRODUÇÃO}

Até bem pouco tempo, o tratamento de águas residuárias restringia-se a destruição de patógenos e preservação do balanço de oxigênio no corpo de água receptor (2). Atualmente existe preocupação não só com a despoluição, mas também com o aproveitamento de nutrientes oriundos de águas residuárias, pela incorporação de biomassa, visando a obtenção de proteínas unicelulares.

* $\quad$ Professora da Universidade Federal do Rio Grande (FURG), Rio Grande, RS.
** $\quad$ Professores da Universidade Federal de Pelotas (UFPel), RS. 
A produção de microrganismos como fonte de proteínas pode ser obtida em grande variedade de substratos, o que torna viável sua produção em diferentes regiões, desde que se escolha, ou se adapte adequadamente o microrganismo ao substrato disponível. Além disso a utilização de resíduos industriais contribui para que se alivie o problema da poluição (11).

O uso de cianobactérias em tratamentos de efluentes industriais vem sendo estudado, visando a valorização de resíduos e água residuárias, com o objetivo de utilizar a biomassa resultante como fertilizante dos solos, ou a aplicação desta proteína de organismos unicelulares para fabricação de ração animal, e mesmo como suplemento da dieta humana.

As cianobactérias representam fonte alternativa de proteína e sua utilização como complemento alimentar já foi estudada em condições experimentais e industriais. A maior parte das experiências disponíveis relaciona-se com a Spirulina $s p$, mas muitas investigações têm sido realizadas com espécies do gênero Oscillatoria (12). Existe porém uma lacuna no que tange a caracterização sensorial desta forma de matériaprima.

Vários autores $(12,7)$ que têm trabalhado com o gênero Ocillatoria afirmam que, apesar de seu teor protéico, esta cianobactéria não tem sido aproveitada para a alimentação humana, em parte por apresentar odor desagradável.

A cianobactéria Aphanothece microscopic Nägeli ocorre em altas concentrações no estuário da Lagoa dos Patos (5). A Lagoa dos Patos é a maior área lacustre do mundo, encontrando-se à margem oeste de sua desembocadura no oceano Atlântico a cidade do Rio Grande (RS). DE LORENZO (6) cita que as florações do gênero Aphanothece são constantes durante todo $\mathrm{o}$ ano e distribuídas nos corpos hídricos adjacentes à cidade do Rio Grande, RS.

Apesar da abundância deste microrganismo nesta região, independente de estudos ambientais e de distribuição $(1,5,6,13)$, não existe qualquer informação quanto ao seu aproveitamento na alimentação humana. Em face disto, o presente trabalho teve como objetivo a caracterização do odor da Aphanothece microscopic Nägeli, visando sua utilização como complemento da dieta alimentar. 


\subsection{MATÉRIA-PRIMA}

Utilizou-se Aphanothece microscopic Nägeli, desidratada por exposição ao sol e pulverizada, com umidade final de aproximadamente $8 \%$. A Spirulina $s p$ e a alga japonesa (em pó) foram obtidas no comércio local. Os padrões de referência utilizados tiveram como base, ração para peixe, bacalhau e ervas frescas.

\subsection{LEVANTAMENTO DE TERMOS}

O levantamento da terminologia foi realizado mediante avaliação de 60 consumidores não orientais e 28 consumidores de origem oriental. Utilizouse uma ficha de tomada de dados, solicitando aos mesmos que descrevessem o odor de três amostras apresentadas (Aphanothece microscopic Nägeli, Spirulina $s p$ e alga japonesa). As amostras foram oferecidas em tubos de ensaio de $1,5 \mathrm{~cm}$ de diâmetro por $15 \mathrm{~cm}$ de comprimento, livres de odor, cobertos com papel alumínio e codificadas com três dígitos aleatórios. Colocou-se chumaço de algodão a $3 \mathrm{~cm}$ do fundo do tubo, como suporte de absorção da amostra portadora de odor e outro distante $3 \mathrm{~cm}$ da extremidade oposta como tampa do tubo.

\subsection{PREPARO DAS AMOSTRAS}

As amostras de padrões de referência pesando em média até $2 \mathrm{~g}$ foram homogenizadas e acrescentadas de $10 \mathrm{~mL}$ de água destilada, em tubos de ensaio fechados. Os mesmos foram centrifugados por 5 minutos para que fossem difundidos os voláteis para a água. Diluições adequadas regularmente perceptíveis foram obtidas para cada amostra.

\subsection{AVALIAÇÃO DO ODOR DA Aphanothece microscopic Nägeli}

\subsubsection{Seleção de julgadores}

A seleção de 19 candidatos foi realizada pelo método proposto por QUEIROZ, TREPTOW \& VIEIRA (14), com base no prisma da classificação de odor de Henning (3). Padrões de referência previamente definidos, que representavam os odores predominantes do produto em função da terminologia descrita, foram apresentados aos candidatos.

Quatro grupos de amostras foram preparadas de maneira bastante uniforme, com diferenças entre os grupos, mas não dentro de cada grupo. As diferenças entre os grupos foram dadas pela classificação de Henning e 
as repetições dentro de cada grupo, em função da igualdade de osmóforos. Os candidatos avaliaram as amostras de iguais osmóforos com cinco repetições. Para avaliação dos resultados partiu-se do princípio que, grande variação entre os grupos de osmóforos e pequena variação entre as repetições dos osmóforos indicam boa habilidade do candidato em distinguir as amostras.

Definiu-se a diferença entre os candidatos a partir da tabela de diferenças significativas de razões de amplitude e média, sugerida por QUEIROZ, TREPTOW \& VIEIRA (14).

Os dados foram coletados através de ficha, utilizando-se escala estruturada de cinco pontos assim definida: 0 - não perceptível; 1 percebe, mas não reconhece; 3 - percebe e reconhece; 5 - percebe, reconhece e descreve.

Os resultados foram tabelados em ordem de dificuldade, em função dos escores obtidos pelos candidatos, estipulado-se os valores, 1 para o grupo mais difícil e 4 para o mais fácil. Desta forma, 1 foi a nota máxima atribuída para o grupo 1; dois para o grupo 2 e assim sucessivamente. Os resultados foram estatisticamente avaliados de acordo com QUEIROZ, TREPTOW \& VIEIRA (14).

\subsubsection{Treinamento de julgadores}

Aos julgadores foram oferecidos os padrões referência em três diluições sucessivas e solicitado que marcassem em escala não estruturada de nove centímetros, o ponto que correspondesse a intensidade do odor percebido. Várias sessões de provas foram realizadas até obter-se reprodutibilidade. Os resultados foram submetidos a análise de variância e ao teste de médias de Tukey ao nível de $5 \%$ de probabilidade (10).

\subsubsection{Avaliação do odor da Aphanothece microscopic Nägeli}

A Aphanothece microscopic Nägeli teve seu odor característico descrito e avaliado, quanto a intensidade e ao fato de ser ou não penetrante, por equipe de 6 julgadores selecionados e treinados, utilizando-se escala não estruturada de nove centímetros (9), conforme Figura 1. Os dados foram estatisticamente analisados pelo teste de Student ao nível de 5\% de probabilidade. 
FIGURA 1 - MODELO DE FICHA DE AVALIAÇÃO

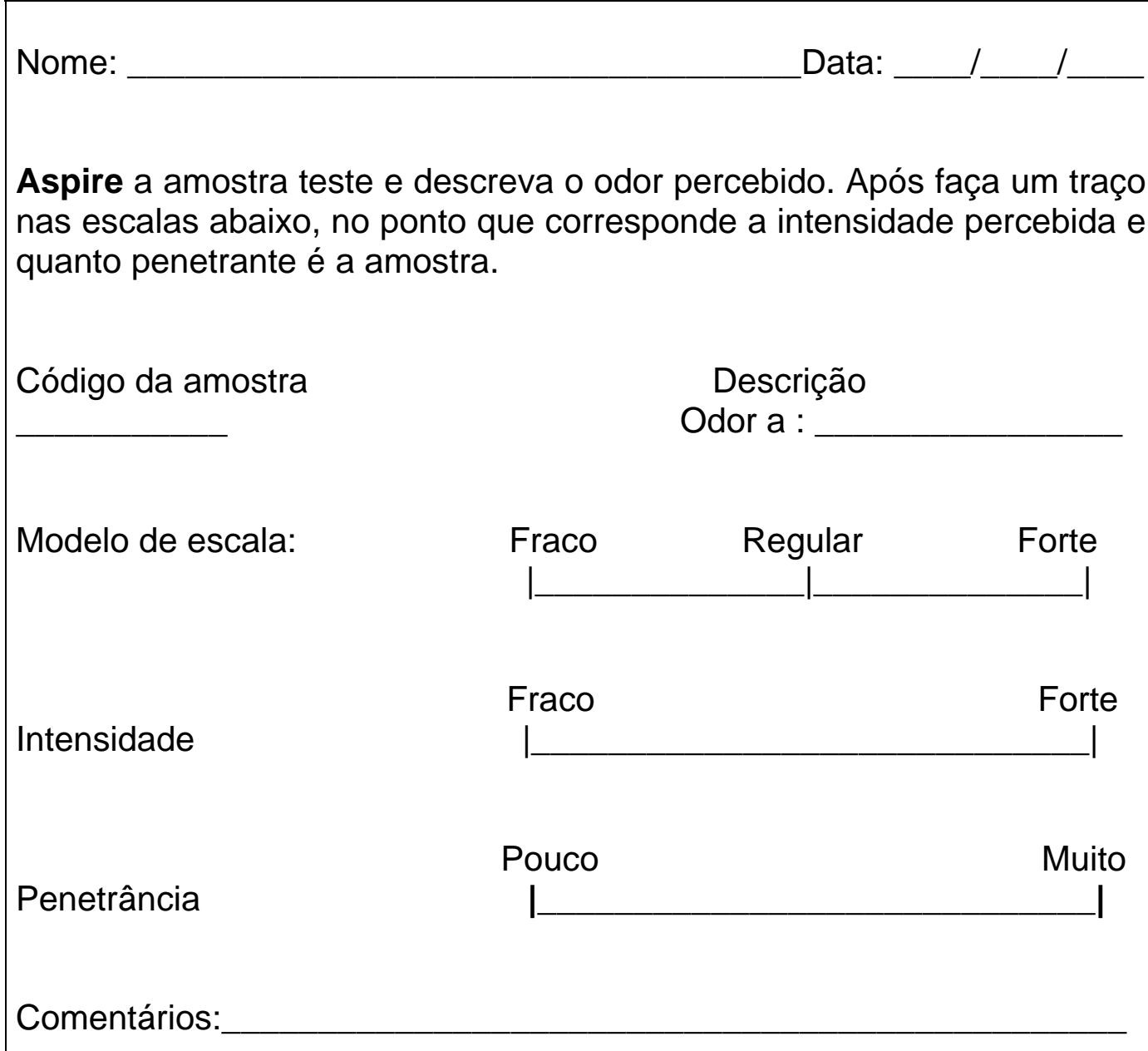

Comentários:

\section{RESULTADOS E DISCUSSÃO}

\subsection{LEVANTAMENTO DA TERMINOLOGIA PARA A DESCRIÇÃO DE} ODORES

A Tabela 1 apresenta a terminologia expressa por 60 consumidores de origem não oriental, em um total de 105 termos para definição do odor da Aphanothece microscopic Nägeli, da Spirulina sp e de uma alga comercial japonesa. 


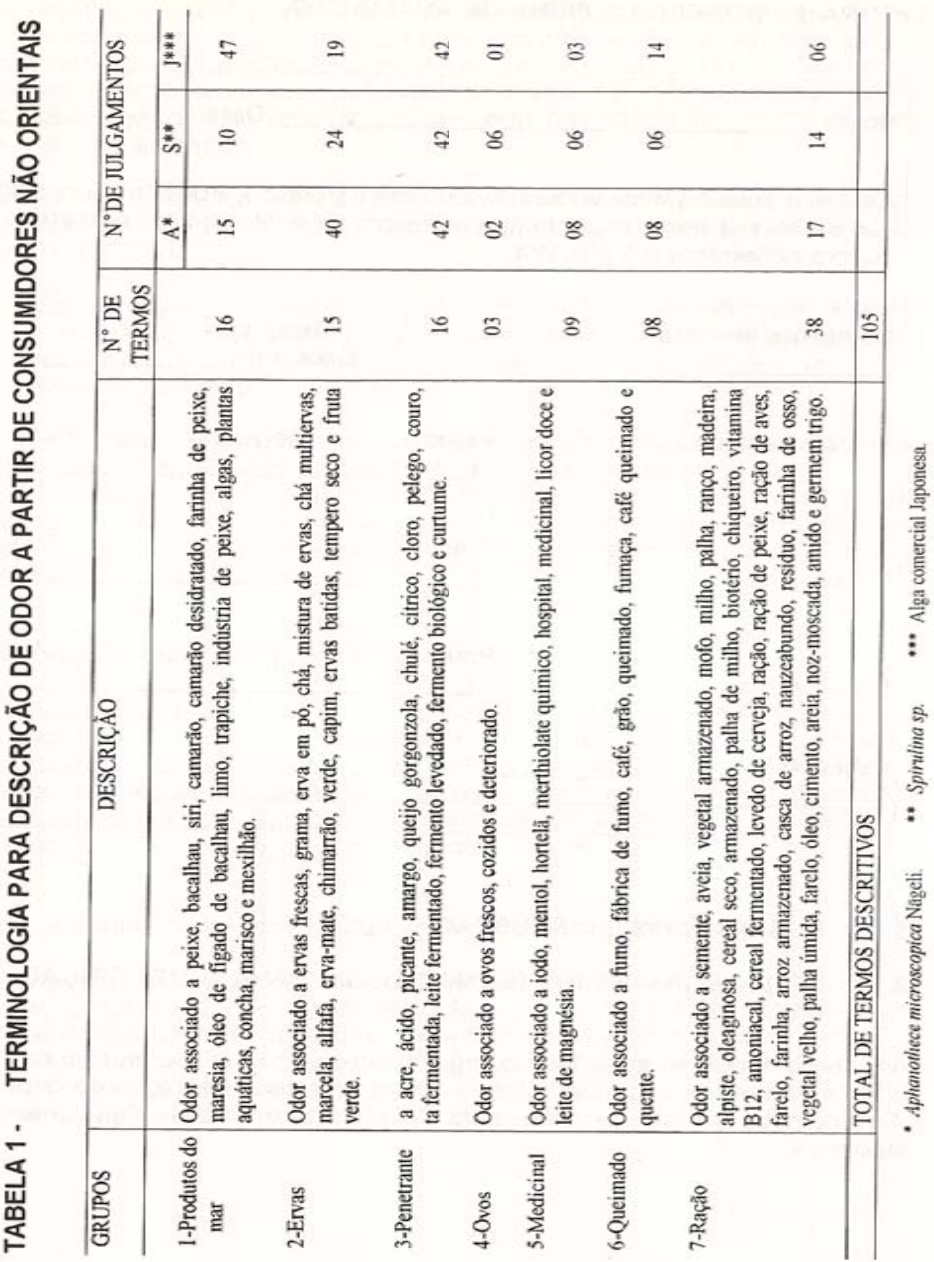

B. CEPPA, Curitiba, v. 16, n. 1, jan.jun. 1998 
É possível observar que os três produtos caracterizam-se por apresentar predominantemente odor penetrante (42 julgamentos), associado ao odor de ervas (40, 24,19 julgamentos), seguido por odor a ração $(17,14,06)$ e a produtos do mar $(15,10$ e 47$)$. Esta constatação pode ser corroborada pela Tabela 2, que expressa a ocorrência da terminologia levantada em percentual, classificada por consenso em quatro grupos: penetrante (composto pelos termos penetrante propriamente dito, ovos, medicinal e queimado) correspondendo ao total de 60 termos descritivos; ervas; ração e produtos do mar. O odor característico da alga japonesa pode ser definido como produtos do mar, perfazendo $78,34 \%$ dos julgamentos.

\section{TABELA 2 - PERCENTUAIS DE OCORRÊNCIA DOS QUATRO GRUPOS BÁSICOS PARA OS TRÊS PRODUTOS EM ANÁLISE}

\begin{tabular}{lccc}
\hline GRUPOS & $\begin{array}{c}\text { Aphanothece } \\
\text { microscopic Nägeli }\end{array}$ & \multicolumn{2}{c}{ PRODUTOS } \\
\cline { 3 - 4 } & & Spirulina sp & Alga Japonesa \\
\hline Penetrante & 100 & 100 & 100 \\
Ervas & 66,67 & 40,00 & 31,67 \\
Ração & 28,33 & 23,33 & 10,00 \\
Produtos do mar & 25,00 & 16,67 & 78,34 \\
\hline
\end{tabular}

Estes resultados demonstram a unanimidade dos consumidores não orientais em descrever o odor das três amostras como penetrante, definindo como odor característico da Aphanothece microscopic Nägeli, o odor a ervas com um percentual de respostas de $66,67 \%$, notadamente superior a Spirulina sp $(40,00 \%)$ e a alga japonesa $(31,67 \%)$.

A Tabela 3 expressa a terminologia desenvolvida por integrantes de uma colônia japonesa do Município de Pelotas, RS. Analisando a relação de termos descritivos verifica-se a diferença cultural e do hábito alimentar entre estes 28 consumidores (15 termos) e os 60 de origem não oriental (105 termos).

Importante salientar que os consumidores de origem oriental foram concordantes em definir as três amostras como alga $(17,20,20$ julgamentos), perfazendo $71,43 \%$ dos termos descritos para Spirulina sp e a alga japonesa e 48,57\% para a Aphanothece microscopic Nägeli.

Analisando as Tabelas 1 e 3 observa-se marcadamente a influência cultural, quando se considera os termos que constituem os grupos penetrante e ração. Os consumidores não orientais apresentaram maior diversidade de termos e inabilidade para expressar a característica de odor de algas como ração. 
TABELA 3 - TERMINOLOGIA PARA DESCRIÇÃO DE ODOR A PARTIR DE CONSUMIDORES DE ORIGEM ORIENTAL

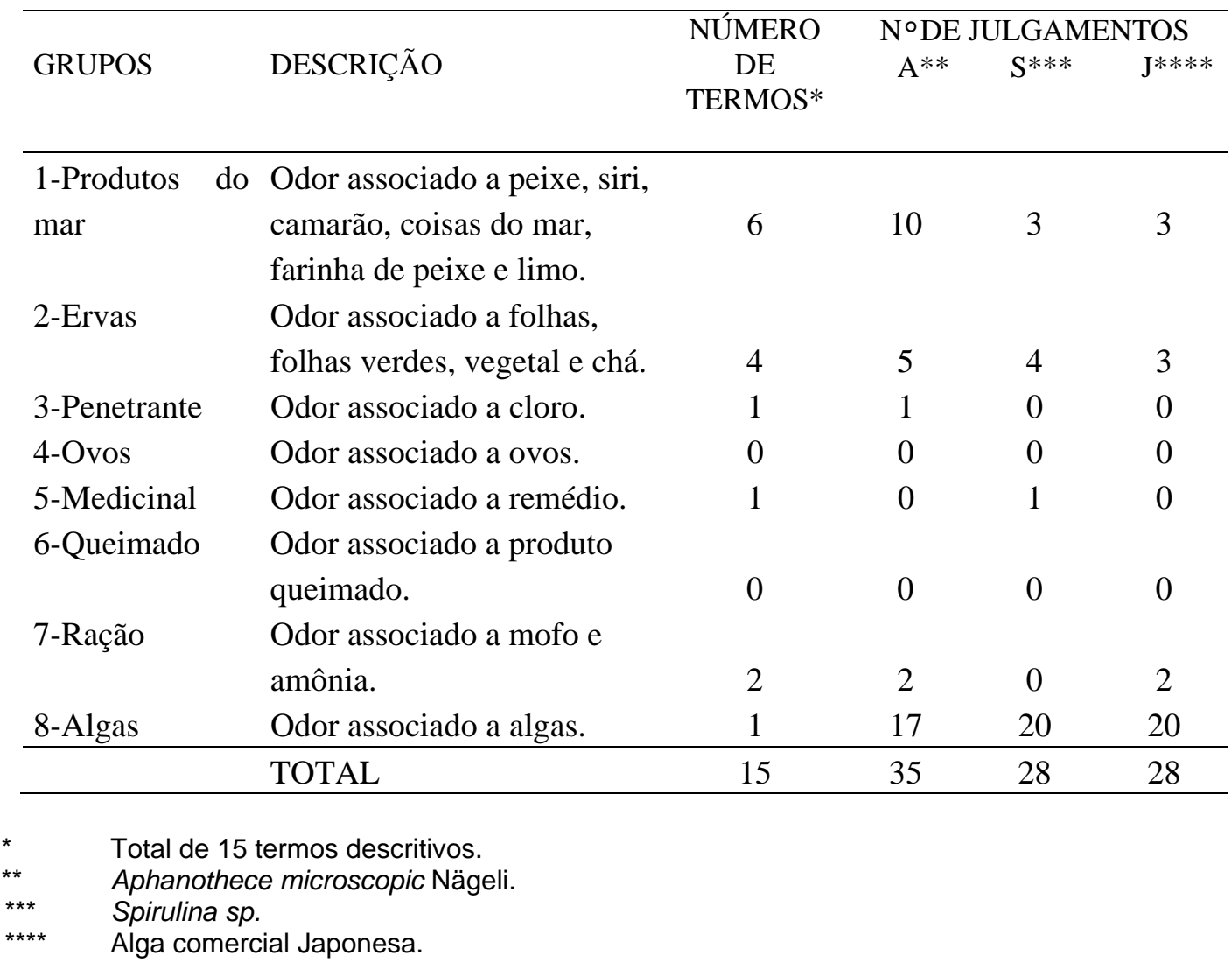

De acordo com FAY (8) as algas quando em grande quantidade apresentam odor pungente. Esta característica de odor descreve compostos que causam sensação de tato na mucosa nasal, como por exemplo cloro e amônia (15), sendo descrita genericamente pela equipe, como penetrante.

$\mathrm{Na}$ Tabela 4 estão registrados os três termos de maior ocorrência, nos quatro grupos mais relevantes, descritos pelos consumidores de origem não oriental.

Em face da maior incidência do termo peixe tomou-se o pescado como padrão de referência para o grupo produtos do mar. Este termo é citado por BRANCO (4) como característico do odor de algas em altas concentrações.

Para o grupo ervas, o termo erva propriamente dito foi o de maior incidência, estando diretamente associado com o verde, ervas frescas e grama, razão pela qual o termo ervas foi escolhido como padrão de referência. 
TABELA 4 - TERMINOLOGIA DEFINIDA PELOS CONSUMIDORES DE ORIGEM NÃO ORIENTAL

\begin{tabular}{llccc}
\hline GRUPOS & \multicolumn{3}{c}{ JULGAMENTOS(\%) } \\
\hline \multirow{2}{*}{ Penetrante } & Penetrante & $\begin{array}{c}\text { Aphanothece } \\
\text { microscopic Nägeli }\end{array}$ & Spirulina sp & $\begin{array}{c}\text { Alga } \\
\text { Japonesa }\end{array}$ \\
& Fumo & 6 & 7 & 14 \\
& Fermento & 6 & 2 & 5 \\
\hline Ervas & Ervas & 3 & 6 & 13 \\
& Erva-mate & 14 & 7 & 9 \\
& Verde & 9 & 1 & 1 \\
\hline Ração & Ração & 6 & 4 & 5 \\
& Farelo & 9 & 3 & 2 \\
\hline Produtos do & Levedo cerveja & 4 & 4 & 0 \\
mar & Peixe & 2 & 2 & 42 \\
& Algas & 11 & 7 & 4 \\
& Maresia & 2 & 3 & 1 \\
\hline
\end{tabular}

Para o grupo ração escolheu-se a ração para peixe, pois este produto representa ao mesmo tempo as características penetrante, ração e produtos do mar.

Considerando que a totalidade dos consumidores definiu o odor dos produtos analisados como penetrante, o cloro foi utilizado como padrão de referência para esta característica.

\subsection{SELEÇÃO DE JULGADORES PARA AVALIAÇÃO DE ODOR}

$\mathrm{Na}$ Tabela 5 estão registrados os dados resultantes da seleção de julgadores, utilizando os osmóforos inerentes aos padrões de referência previamente estabelecidos.

Esta Tabela permite visualizar a ordem de dificuldade dos candidatos quanto a habilidade de perceber e descrever odores. É possível verificar que o grupo descrito como ração foi o mais difícil de ser definido, em função da diversidade de termos levantados (Tabela 1 ).

A característica penetrante também foi de fácil definição, quando comparada ao grupo ração. Este termo está associado ao odor característico da Aphanothece microscopic Nägeli, descrito como penetrante propriamente dito, independente da definição de ervas, produtos de mar ou ração. Esta característica quando não acrescida dos termos que constituem os grupos 4,5 e 6 (Tabela 1 ) representa um total 
de 16 termos. Assim os valores máximos tabulados, expressos na Tabela 5, correspondem a: 1 grupo ração; 2 grupo ervas; 3 grupo penetrante e 4 grupo produtos do mar.

\section{TABELA 5 - OSMÓFOROS REFERÊNCIA E SOMATÓRIO TOTAL DE NOTAS EXPRESSAS PELOS CANDIDATOS *}

\begin{tabular}{llc}
\hline GRUPOS & PRODUTOS & SOMATÓRIO-NOTAS \\
\hline Ração & ração de peixe & \\
& germem de trigo & 140 \\
& levedo - cerveja & \\
& vitamina B12 & \\
& Aphanothece microscopic Nägeli & \\
\hline Ervas & erva mate & 300 \\
& Grama & \\
& Multiervas & \\
& Marcela & \\
& Aphanothece microscopic Nägeli & \\
\hline Penetrante & Cloro & \\
& Iodo & \\
& Amônia & \\
& Fumo & \\
& Aphanothece microscopic Nägeli & \\
\hline Produtos do mar & peixe fresco & \\
& Bacalhau & \\
& ração peixe & \\
& Mexilhão \\
Aphanothece microscopic Nägeli & \\
\hline
\end{tabular}

* 19 candidatos.

A Tabela 6 fornece a análise dos valores atribuídos pelos candidatos e expresso como média e razão de amplitude para os dados tabulados em ordem de dificuldade.

A avaliação destes dados permitiu eliminar, a princípio, os candidatos 1 e 16 por apresentarem razões de amplitude menores que a unidade. Os candidatos que apresentaram maiores razões de amplitude foram: 5, 10, 12 e 18, com médias correspondentes a 2,21; 1,84; 2,12 e 2,19, respectivamente.

Foram selecionados doze candidatos comparando-se as diferenças de média e a razão de amplitude com as do candidato 5, que apresentou a maior média em valores absolutos (Tabela 6). 


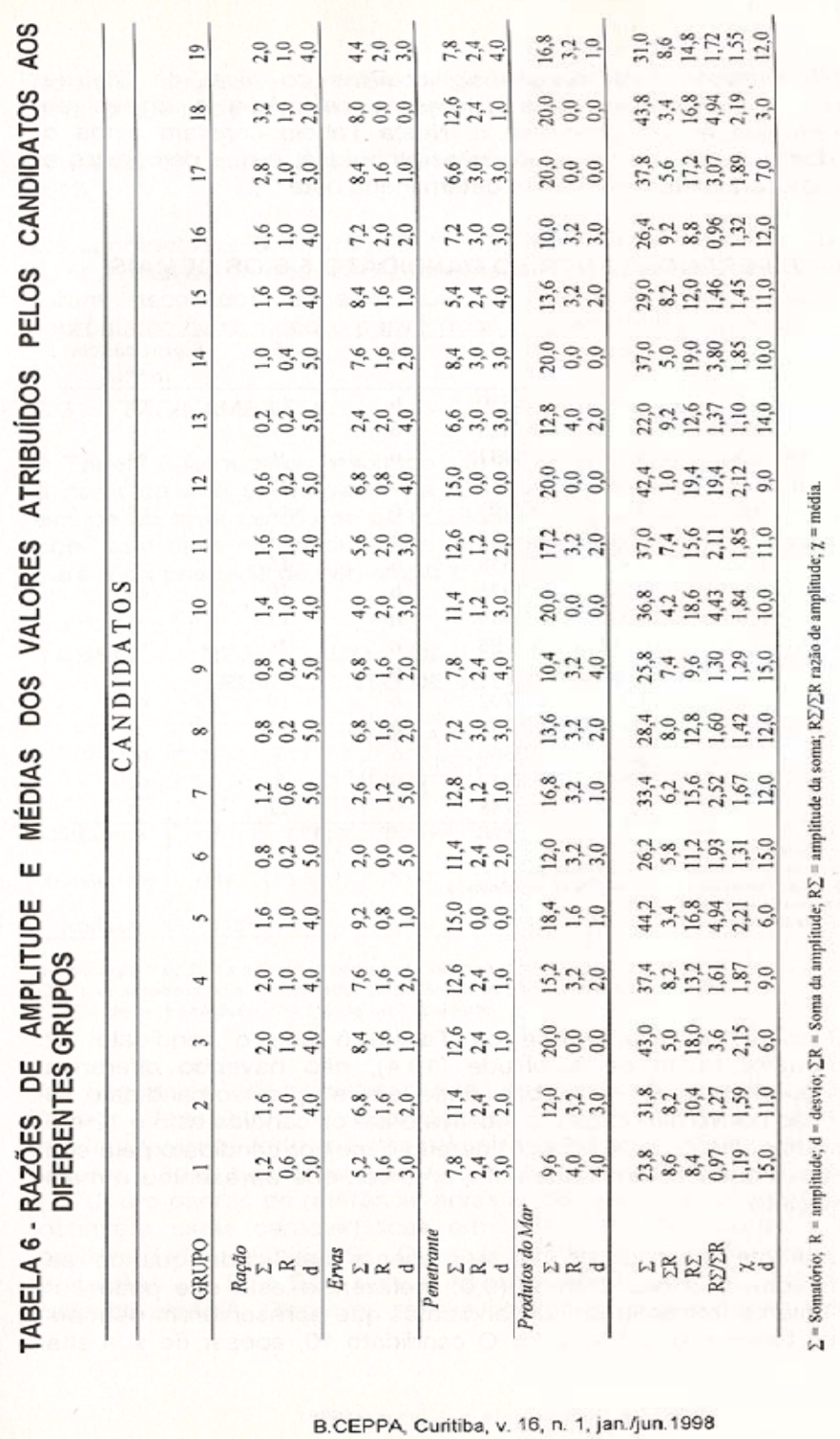

65 
A Tabela 7 fornece as diferenças significativas ao nível de $5 \%$ de probabilidade entre os candidatos, diferenças de média e razões de amplitude em relação ao candidato 5 . Nesta Tabela constam ainda o somatório dos desvios das notas por estes atribuídas, o que demonstra a habilidade dos candidatos em manter determinada nota.

\section{TABELA 7 - DIFERENÇAS ENTRE O CANDIDATO 5 E OS DEMAIS}

\begin{tabular}{lcccccc}
\hline $\begin{array}{l}\text { Diferenças } \\
\text { Candidatos }\end{array}$ & 1 & 2 & 3 & 4 & 5 & $\begin{array}{c}\text { Significância } \\
(\alpha 5 \%)\end{array}$ \\
\hline $5^{\circ} \mathrm{e} 2^{\circ}$ & 0,62 & 3,66 & 0,6650 & 6 & 11 & $\mathrm{~ns}$ \\
$5^{\circ} \mathrm{e} 3^{\circ}$ & 0,06 & 1,33 & 0,7623 & 6 & 6 & $\mathrm{~ns}$ \\
$5^{\circ} \mathrm{e} 4^{\circ}$ & 0,34 & 3,32 & 0,6831 & 6 & 9 & $\mathrm{~ns}$ \\
$5^{\circ} \mathrm{e} 6^{\circ}$ & 0,90 & 3,00 & 0,6883 & 6 & 15 & $\mathrm{~s}$ \\
$5^{\circ} \mathrm{e} 7^{\circ}$ & 0,54 & 2,41 & 0,7103 & 6 & 12 & $\mathrm{~ns}$ \\
$5^{\circ} \mathrm{e} 8^{\circ}$ & 0,79 & 3,33 & 0,6789 & 6 & 12 & $\mathrm{~s}$ \\
$5^{\circ} \mathrm{e} 9^{\circ}$ & 0,92 & 3,63 & 0,6638 & 6 & 15 & $\mathrm{~s}$ \\
$5^{\circ} \mathrm{e} 10^{\circ}$ & 0,37 & 0,50 & 0,7926 & 6 & 10 & $\mathrm{~ns}$ \\
$5^{\circ} \mathrm{e} 11^{\circ}$ & 0,36 & 2,82 & 0,6949 & 6 & 11 & $\mathrm{~ns}$ \\
$5^{\circ} \mathrm{e} 12^{\circ}$ & 0,09 & 14,47 & 0,4293 & 6 & 9 & $\mathrm{~ns}$ \\
$5^{\circ} \mathrm{e} 13^{\circ}$ & 1,11 & 3,56 & 0,6768 & 6 & 14 & $\mathrm{~s}$ \\
$5^{\circ} \mathrm{e} 14^{\circ}$ & 0,36 & 1,13 & 0,7702 & 6 & 10 & $\mathrm{~ns}$ \\
$5^{\circ} \mathrm{e} 15^{\circ}$ & 0,76 & 3,47 & 0,6732 & 6 & 11 & $\mathrm{~s}$ \\
$5^{\circ} \mathrm{e} 17^{\circ}$ & 0,32 & 1,86 & 0,7360 & 6 & 7 & $\mathrm{~ns}$ \\
$5^{\circ} \mathrm{e} 18^{\circ}$ & 0,02 & 0,01 & 0,8049 & 6 & 3 & $\mathrm{~ns}$ \\
$5^{\circ} \mathrm{e} 19^{\circ}$ & 0,66 & 3,21 & 0,6788 & 6 & 12 & $\mathrm{~ns}$ \\
\hline
\end{tabular}

1 = Diferença de média entre candidatos.

2 = Diferença de razão de amplitude entre candidatos

3 = Diferença de média tabelada ao nível de $5 \%$ de significância.

4 = Somatório dos desvios do candidato 5 .

5 = Somatório dos desvios dos candidatos.

ns = não significativo.

$\mathrm{s}=$ significativo.

É possível constatar pela análise da Tabela 6 que o candidato 12 apresentou maior razão de amplitude $(19,4)$, não havendo diferença significativa ao nível de $5 \%$ de probabilidade, em relação ao candidato 18 (Tabela 7). Não houve diferença significativa entre os candidatos 5 e 12 e 5 e 18, porém este último pode ser considerado o melhor candidato pela sua habilidade em manter determinada nota, uma vez que apresentou o mais baixo desvio $(3,0)$.

Comparativamente o candidato 12 teria menor habilidade quando se considera a somatória dos desvios $(9,0)$, deficiência esta que pode ser corrigida mediante treinamento. Os candidatos que apresentaram os mais altos desvios foram o 6, o 9 e o 13. O candidato 10, apesar de sua alta 
razão de amplitude apresentou baixa habilidade em repetir determinada nota, o que pode também ser superado pelo treinamento.

A análise dos dados sugeriu a eliminação dos candidatos 1, 6, 8, 9, 13, 15 e 16.

Os candidatos $2,3,4,5,7,10,11,12,14,17,18$ e 19 foram selecionados para avaliação do odor da Aphanothece microscopic Nägeli. Dentre estes, foram escolhidos aqueles que demonstraram maior habilidade na reprodução de resultados para formar a equipe de 6 julgadores.

\subsection{TREINAMENTO}

A Tabela 8 fornece os resultados do treinamento da equipe de julgadores, a partir da análise de variância. Os dados demonstram uniformidade da equipe ao nível de $5 \%$ de probabilidade para a medida de intensidade de odor com base nos padrões de referência (ração, peixe, cloro e ervas) e para a propriedade de penetrância.

\section{TABELA 8 - RESULTADO DA ANÁLISE DE VARIÂNCIA DO TREINAMENTO DE JULGADORES}

\begin{tabular}{|c|c|c|c|c|c|c|c|c|}
\hline \multicolumn{7}{|c|}{ INTENSIDADE } & \multicolumn{2}{|c|}{ PENETRÂNCIA } \\
\hline & \multicolumn{2}{|c|}{ FRACO } & \multicolumn{2}{|c|}{ MÉDIO } & \multicolumn{2}{|c|}{ FORTE } & & \\
\hline & $\mathrm{QM}$ & $\mathrm{F}$ & QM & $\mathrm{F}$ & $\mathrm{QM}$ & $\bar{F}$ & $\mathrm{QM}$ & $\mathrm{F}$ \\
\hline Julgadores & 0,03 & $0,71 \mathrm{~ns}$ & 0,201 & $2,21 \mathrm{~ns}$ & 0,25 & $1,00 \mathrm{~ns}$ & 0,312 & $1,33 \mathrm{~ns}$ \\
\hline Tratamentos & 0,16 & $3,81 *$ & 2,513 & $27,61 * *$ & 0,42 & $1,68 \mathrm{~ns}$ & 12,74 & $54,44 * *$ \\
\hline Resíduo & 0,042 & & 0,091 & & 0,25 & & 0,234 & \\
\hline
\end{tabular}

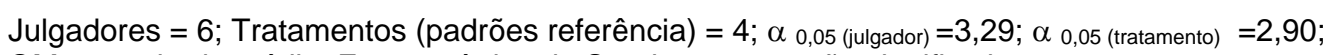
$\mathrm{QM}=$ quadrado médio; $\mathrm{F}=$ estatística de Snedecor; $\mathrm{ns}=$ não significativo;

${ }^{*}=5 \%$ de probabilidade, ${ }^{* *}=1 \%$ de probabilidade.

Os julgadores descreveram o odor da Aphanothece microscopic Nägeli (sua intensidade e penetrância), como sendo característico, moderadamente forte a ervas e ligeiramente penetrante. Comparando os valores médios de intensidade de odor da Aphanothece microscopic Nägeli $(7,70)$ e o padrão de referência ervas $(7,38)$ verificou-se que os julgadores definiram estas características em igual nível de intensidade (5\% de probabilidade) (Tabela 9). O mesmo ocorreu com a propriedade de penetrância, 2,50 e 2,38 para a Aphanothece microscopic Nägeli e ervas, respectivamente. Observou-se similar dispersão dos dados em torno da 
média, o que se traduz em homogeneidade da equipe, conforme já constatado no item 3.3.

\title{
TABELA 9 - COMPARAÇÃO DE MÉDIAS PELO TESTE DE STUDENT PARA AS CARACTERÍSTICAS DE INTENSIDADE E PENETRÂNCIA DA Aphanothece microscopic Nägeli E ERVAS
}

\begin{tabular}{c|c|c|c|c}
\hline \multicolumn{3}{c|}{ INTENSIDADE } & \multicolumn{2}{c}{ PENETRÂNCIA } \\
\hline Estatísticas & $\begin{array}{c}\text { Aphanothece } \\
\text { microscopic } \\
\text { Nägeli }\end{array}$ & Ervas & $\begin{array}{c}\text { Aphanothece } \\
\text { microscopic } \\
\text { Nägeli }\end{array}$ & Ervas \\
\hline $\mathrm{X}$ & 7,70 & 7,38 & 2,50 & 2,38 \\
\hline $\mathrm{S}$ & 7,69 & 7,37 & 0,48 & 0,37 \\
\hline $\mathrm{Tc}$ & \multicolumn{2}{c|}{$0,17 \mathrm{~ns}$} & \multicolumn{2}{c}{$0,32 \mathrm{~ns}$} \\
\hline $\mathrm{Tt}$ & 2,23 & 2,23 \\
\hline
\end{tabular}

$X=$ média; $\mathrm{S}=$ desvio padrão; $\mathrm{T}=$ estatística; $\mathrm{Tc}=\mathrm{T}$ calculado; $\mathrm{Tt}=\mathrm{T}$ tabelado $\left(\alpha_{0,05 \%} 10 \mathrm{gl}.\right) \mathrm{ns}=$ não significativo.

\section{CONCLUSÃO}

A Aphanothece microscopic Nägeli, a Spirulina e a alga comercial japonesa caracterizam-se por apresentar odor penetrante, associado em primeiro lugar com ervas, em segundo com odor de ração e finalmente com produtos do mar.

Consumidores com descendência oriental foram mais objetivos na descrição do odor das três espécies avaliadas.

A Aphanothece microscopic Nägeli apresenta odor característico, moderadamente forte a ervas e ligeiramente penetrante.

\begin{abstract}
The attribute odor of Aphanothece microscopic Nägeli has been characterized aiming its application into human feeding. The terminology was addressed with the help of consumers, comparing it to the Spirulina $s p$ and to a commercial seaweed. The team was selected and trained to express the intensity of the scent, in agreement with standards of penetrating, herbs, ration, and sea products, obtained from the terminology described by consumers. The results indicated that both Aphanothece microscopic Nägeli and Spirulina $s p$, as well as the commercial Japanese seaweed, present penetrating scent as predominant characteristic, followed in order by herbal, ration, and sea-product scent. The Aphanothece microscopic Nägeli carries a moderately strong herbal scent and a lightly penetrating characteristic.
\end{abstract}




\section{REFERÊNCIAS BIBLIOGRÁFICAS}

1 ABREU, P.C.O.V. Variações temporais de biomassa fitoplantônica (clorofila) e relação com os fatores abióticos no canal de acesso ao estuário da Lagoa dos Patos. Rio Grande, 1987. 107 p. Dissertação (Mestrado), Curso de PósGraduação em Oceanografia Biológica, Universidade Federal do Rio Grande.

AKHTAR, Y., GRAFIAR, A. Removal of $\mathrm{NH}_{3}-\mathrm{N}$ from domestic wastewater by fungy. Biotecnology, v. 8, n. 8, p. 601-4, 1986.

AMERINE, M.A., PANGBORN, R.M., ROESSLER, E.B. Principles of sensory evaluation of foods. New York: Academic Press, 1965. $605 \mathrm{p}$.

BRANCO, S.M. Hidrobiologia aplicada a engenharia sanitária. 3.ed. São Paulo : CETESB/ASCETESB, 1986. 616 p.

5 COUTINHO, R. Taxinomia, distribuição, crescimento sazonal e produção de algas bentônicas na Lagoa dos Patos, RS. Rio Grande, 1992. 232 p. Dissertação (Mestrado), Curso de PósGraduação em Oceanografia Biológica, Universidade Federal do Rio Grande.

DE LORENZO, Mariangela. Relação de nutrientes e de fatores ambientais na ocorrência de cianobactérias Aphanothece $s p$ ao redor da cidade de Rio Grande. Rio Grande, 1995. 25 p. Trabalho de Graduação, Curso de Oceanografia, Área de Gerenciamento Costeiro, Universidade Federal do Rio Grande.

FAINTUCH, B.L., SATO, S., AQUARONE, E. Emprego de algumas fontes nitrogenadas na obtenção de biomassa de Oscillatória limnetica. Rev. Microbiol., v. 23, n. 1, p. 32-6, 1992.

8 FAY, Peter. The blue-greens (Cyanophyta - Cyanobacteria). Maryland: Edward Arnold, 1983. 88 p.

9 GATCHALIAN, M.M. Sensory evaluation methods with statistical analysis. Quezon : Universidade das Filipinas, 1981. 420 p.

10 GOMES, F.P. Curso de estatística experimental. 12.ed. Piracicaba : Nobel, 1987. $430 \mathrm{p}$.

11 LIMA, V., AQUARONE, E., BORZANI, W. Biotecnologia: tecnologia das fermentações. São Paulo : Edgar Blucher, 1986. 243 p. 
12 OREN, A., PADAN, E. Induction of anaerobic, foto autotrofie growth in the cyanobacterium Oscillatoria limnetic. J. Bacteriol., v. 133, p. 358-63, 1987.

13 PROENÇA, L.A. Ciclo anual da produção primária, biomassa do fitoplancto e carbono orgânico particulado em áreas rasas da porção sul da Lagoa dos Patos. Rio Grande, 1986. 89 p. Tese (Mestrado), Curso de Pós-Graduação em Oceanografia Biológica, Universidade Federal do Rio Grande.

14 QUEIROZ, M.I., TREPTOW, R.O., VIEIRA, H. Seleção de julgadores para avaliação de odor. In: ENCONTRO NACIONAL DE ANALISTAS DE ALIMENTOS, 8., Porto Alegre, 1993. Anais... Porto Alegre, 1993. 1 p. (TI 065).

15 QUEIROZ, M.I. Introdução a análise sensorial. Campinas :

Faculdade de Engenharia de Alimentos, UNICAMP, 1984. $193 \mathrm{p}$. (Mimeografado). 